\title{
Infection Rates and Healing Using Bone Wax and a Soluble Polymer Material
}

\author{
Tadeusz Wellisz MD, Yuehuei H. An MD, Xuejun Wen MD, PhD, \\ Qian Kang MD, Christopher M. Hill VMD, Jonathan K. Armstrong PhD
}

Received: 17 June 2007/ Accepted: 29 October 2007

(C) The Association of Bone and Joint Surgeons 2008

\begin{abstract}
The effects of using a newly available watersoluble polymer bone hemostatic material in a contaminated environment were assessed in a rabbit tibial defect model. Infection rates and healing of polymer-treated bone were compared with the infection and healing of bone waxtreated bone and untreated controls after a bacterial challenge. Defects created in 24 rabbit tibias were treated with the polymer or bone wax, or left without a hemostatic agent. The defects were inoculated with Staphylococcus aureus ATCC-29213 $\left(2.5 \times 10^{4}\right.$ colony-forming units). After 4 weeks, all defects treated with bone wax were infected and osteomyelitis had developed, and none had evidence of bone healing. In the polymer and control groups, two defects in each group (25\%) had osteomyelitis
\end{abstract}

Two of the authors (Tadeusz Wellisz, Jonathan K. Armstrong) have a financial interest in Ceremed, Inc. Four of the authors (Yuehuei H. An, Xuejun Wen, Qian Kang, Christopher M. Hill) received grant support from Ceremed, Inc to support this research.

Each author certifies that his or her institution has approved the animal protocol for this investigation and that all investigations were conducted in conformity with ethical principles of research.

T. Wellisz $(\square)$

Division of Plastic and Reconstructive Surgery, the Department of Neurosurgery, University of Southern California, 536 S Rimpau Blvd, Los Angeles, CA 90020, USA

e-mail: tadeusz@wellisz.com

Y. H. An, X. Wen, Q. Kang, C. M. Hill

Orthopaedic Research Laboratory, Department of Orthopaedic Surgery, Medical University of South Carolina,

Charleston, SC, USA

\section{J. K. Armstrong}

Department of Physiology and Biophysics,

Keck School of Medicine, University of Southern California,

Los Angeles, CA, USA develop. The remaining six defects in each group (75\%) showed no osteomyelitis and exhibited normal bone healing. The polymer-treated defects had a considerably lower rate of osteomyelitis and positive bone cultures compared with the bone wax-treated group. There were no differences between the polymer-treated and control groups in the rates of osteomyelitis, positive cultures, or bone healing. The use of a soluble polymer as an alternative to bone wax may decrease the rates of postoperative bone infections.

\section{Introduction}

Bone wax, which largely is composed of beeswax, is widely used for bone hemostasis. Unadulterated beeswax was used for amputation hemostasis during the US Civil War. The development of modern bone wax has been attributed to Horsley in 1892 [18, 30]. Currently available formulations of bone wax have not changed much and are comprised of water-insoluble beeswax softened with paraffin and/or isopropyl palmitate [33]. Bone wax has no inherent hemostasis quality; its effect is to tamponade the vascular spaces in the bone. Although effective in stopping bone bleeding, bone wax has numerous troublesome adverse effects [41]. Once applied to bone, bone wax remains at the site indefinitely. Bone wax is known to increase infection rates, interfere with bone healing, and elicit chronic inflammatory reactions [33]. Continued use of bone wax for bone hemostasis, despite its known adverse effects, may be partly the result of the absence of a suitable alternative.

A new synthetic bone hemostasis material made of water-soluble alkylene oxide copolymers recently became commercially available [41]. The use of a water-soluble 
synthetic wax for bone hemostasis comprised of similar alkylene oxide copolymers was first described by Wang et al. [40]. These copolymers have a long history in the medical and pharmaceutical fields [13, 40]. They are considered inert because they are eliminated from the body unchanged without being metabolized $[10,16,21]$. Because these copolymers are hydrophilic, they stick well to wet surfaces and thus are well suited for bone hemostasis.

Our animal study was designed to determine the behavior of these two bone hemostasis materials in a contaminated environment. Tibial bone defects received a bacterial challenge after they were either treated with the polymer material or bone wax or left untreated as a control. The first objective of this study was to determine if the use of the polymer material affected the infection rates; the second objective was to determine if the polymer material affected healing of bone defects in a contaminated environment.

\section{Materials and Methods}

Cortical bone defects created in a rabbit tibial defect model were treated in one of three ways. The edges of the defects in the first group of animals were coated with a commercially available blend of water-soluble alkylene oxide copolymers (Ostene; Ceremed, Inc, Los Angeles, CA). The U.S. Food and Drug Administration (FDA) has cleared this material for use as an implant and for control of bleeding from bone surfaces. The second group received a coating of bone wax, a beeswax-based hemostat (Bone Wax; Ethicon, Inc, Somerville, NJ). The defects in the third group were used as controls and were not treated with a hemostatic agent.

All procedures were approved by the Institutional Animal Care and Use Committee at the Medical University of South Carolina. Twenty-four female New Zealand White rabbits $(2.75 \pm 0.025 \mathrm{~kg})$ were randomly assigned to one of three groups ( $\mathrm{n}=8$ per group). Buprenorphine $(0.02 \mathrm{mg} / \mathrm{kg})$ was administered before surgery; the rabbits were anesthetized using $30 \mathrm{mg} / \mathrm{kg}$ ketamine, $5 \mathrm{mg} / \mathrm{kg}$ xylazine, and 1 to $3 \mathrm{mg} / \mathrm{kg}$ atropine intramuscularly and maintained on isoflurane after intubation. Surgery was performed using standard aseptic techniques. Animals from each group were included at each laboratory session with the same operators performing all surgeries. The rabbit's right hind limb was shaved and the skin cleaned with a solution containing $7.5 \%$ povidone-iodine and $70 \%$ isopropyl alcohol. Without the use of a tourniquet, a $2.0-\mathrm{cm}$ anteromedial incision was made to access the proximal tibia. A cortical window measuring $4 \mathrm{~mm} \times 12 \mathrm{~mm}$ was created at the anteromedial facet of the proximal tibia using a 4.0-mm drill bit and a microoscillating saw under constant irrigation. In the study groups, $0.25 \mathrm{~g}$ of material was applied to the edges of the cortical bony defects. An inoculum of Staphylococcus aureus strain ATCC-29230 $\left(2.5 \times 10^{4}\right.$ colony-forming units in $0.1 \mathrm{~mL}$ saline $)$ was introduced into the intramedullary canal through the defect. The organisms had been grown overnight in tryptic soy broth at $37^{\circ} \mathrm{C}$ assuring confluent growth, washed twice in phosphate-buffered saline, and resuspended in a balanced salt solution. The plate count method was used to confirm consistent bacterial inoculum load for all defects. The wound was closed in layers using monofilament sutures and the incision was covered with sterile dressing. After surgery, the animals were given buprenorphine $(0.02$ $0.05 \mathrm{mg} / \mathrm{kg}$ ) as needed every 12 hours.

Four weeks after surgery, the animals were euthanized using an intravenous overdose of pentobarbital. Radiographs of the tibias were taken. The tibias were exposed through the original incisions under sterile conditions, and bone was swabbed for bacterial culture and typing. The tibias were harvested and cut into two segments using an oscillating saw through the center of the original cortical defect. The upper part of the bone explant was cultured. Cultures were grown overnight in $5 \mathrm{~mL}$ of tryptic soy broth media. A 1-mL aliquot of the culture was removed, centrifuged to remove the growth medium, and diluted by $10^{6}$ with phosphate-buffered saline. One milliliter of the diluted culture was plated on standard agar plates and colonies were counted after 24 hours. Growth was graded as follows: no growth; less than 20 colonies were graded light growth; 20 to 80 colonies were graded moderate growth; and more than 80 colonies were graded heavy growth.

The lower part of the tibial bone and surrounding soft tissue were fixed in $10 \%$ buffered formalin, decalcified, and processed for paraffin sectioning. Sections were examined microscopically after hematoxylin and eosin staining. Image scanning of sections was performed using a ScanScope XT System (Aperio Technologies Inc, Vista, CA) at $\times 20$ magnification courtesy of the Tissue Procurement Core Laboratory (UCLA School of Medicine, Los Angeles, CA). All radiographs and sections were viewed independently by two observers (TW, XW) who were blinded to the results.

For the infection and bone healing research questions, the study had three treatment arms: polymer, bone wax, and control. The results for the first question were categorized as either infected or not infected. The results for the second question were categorized as either healing or not healing. Statistical analysis of the data was performed on the $3 \times 2$ contingency table using the Fisher-FreemanHalton exact test [27]. A value of $p<.05$ was considered significant. 


\section{Results}

Animals that received the water-soluble polymer and animals in the control group showed a significantly lower incidence of osteomyelitis ( $\leq 0.004)$, and positive bone cultures $(\mathrm{p} \leq 0.02)$, compared with the bone wax-treated group (Fig. 1). The polymer had no effect on the infection rate and rate of positive cultures compared with controls $(\mathrm{p} \leq 0.001)$. At 4 weeks, all of the animals in the bone wax group (eight of eight) had radiographic evidence of moderate to severe osteomyelitis, including periosteal reaction and bone lysis (Fig. 2). On histologic examination, all of the bone wax group specimens exhibited typical signs of bone infection: development of abscess lesions, destruction of cortical bone, and periosteal reaction. The bone marrow structure was destroyed in all those specimens (Fig. 3). By comparison, six of the eight animals had normal radiographs in the polymer (Fig. 2B) and the control groups (Fig. 2C); the other two animals in each group had radiographic evidence of osteomyelitis together with typical histologic signs of bone infection, including abscesses, and destruction of bone and marrow structures.

Cultures of the swabs and bone segments were positive for the inoculated strain of Staphylococcus aureus in 100\% of the bone wax-treated specimens. In the polymer group and the control group, cultures from the animals with radiographic evidence of osteomyelitis were positive, and one additional animal of the six with normal radiographs in each group had a positive culture; five animals in each group had no evidence of infection.

The use of the water-soluble polymer did not affect bone healing compared with controls $(p \leq 0.001)$. All of the cortical defects in the animals without radiographic

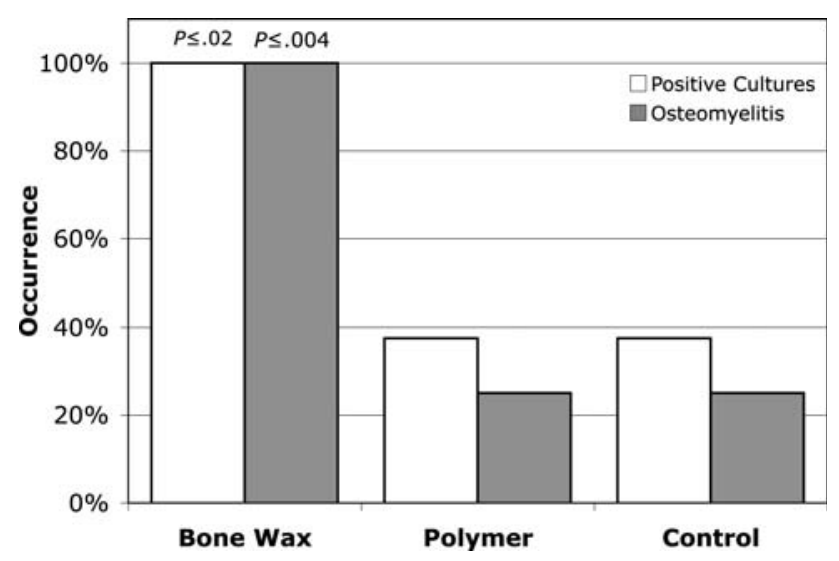

Fig. 1 The application of the water-soluble polymer to a cortical defect significantly decreased the rate of osteomyelitis formation $(p \leq 0.004)$ and rates of positive cultures $(p \leq 0.02)$ compared with bone wax. There was no difference between the polymer group and the untreated control group in the rates of osteomyelitis, positive cultures, or healing of bone defects $(\mathrm{p} \leq 0.001)$. evidence of infection had histologic evidence of bone healing. In the polymer group, five of the cortical defects had been closed by new bone formation (Fig. 4) and one was partially closed. In the control group, four of cortical defects were closed (Fig. 5) and two were partially closed. None of the defects with radiographic verification of infection had evidence of healing in any treatment group.

\section{Discussion}

The rabbit tibial model has been used to study proposed treatments for osteomyelitis [1] and provides a practical means to investigate whether the use of a water-soluble bone hemostasis material in a contaminated environment might be less likely to promote the development of osteomyelitis than bone wax, and secondarily whether the polymer material might influence bone healing. Some limitations of this study are that, like with most animal studies, there is no certainty that the findings are predictive of the likely outcome in a human subject. Also, the type of bacteria and the method of application do not necessarily reflect the typical clinical situation.

In several animal studies, bone wax was shown to increase infection rates and impair the ability of bone to clear bacteria [22, 28, 31]. In a rabbit study, the cancellous bone of the iliac crest was inoculated with Staphylococcus aureus followed by placement of either bone wax or a steel rod. The authors concluded that bone wax impaired the ability of cancellous bone to clear the infection [22]. In a rat tibia model, the presence of bone wax reduced the amount of bacteria needed to produce Staphylococcus aureus osteomyelitis by a factor of 10,000 [28]. In a retrospective clinical study, infection rates after spinal surgery were assessed during a 3-month period [15]. Surgical site infections occurred in six of 42 cases $(14.3 \%)$ in which bone wax was used and in only one of 72 cases (1.4\%) in which it was not used.

There have been no clinical reports or in vivo studies published to date reporting complications or infections with the use of the polymer material evaluated in this study. One in vitro study involving one of the component polymers (poloxamer 188) showed coating silicone wafers with the polymer reduced bacterial adhesion and was more effective than iodine in reducing Staphylococcus epidermidis colony counts on silicone surfaces [25]. In our study, the use of the polymer material considerably reduced the infection rate compared with the use of bone wax, and it had no effect on the infection rate compared with the untreated controls.

The propensity to interfere with bone healing is a wellknown property of bone wax [41]. In the 1924 edition of 

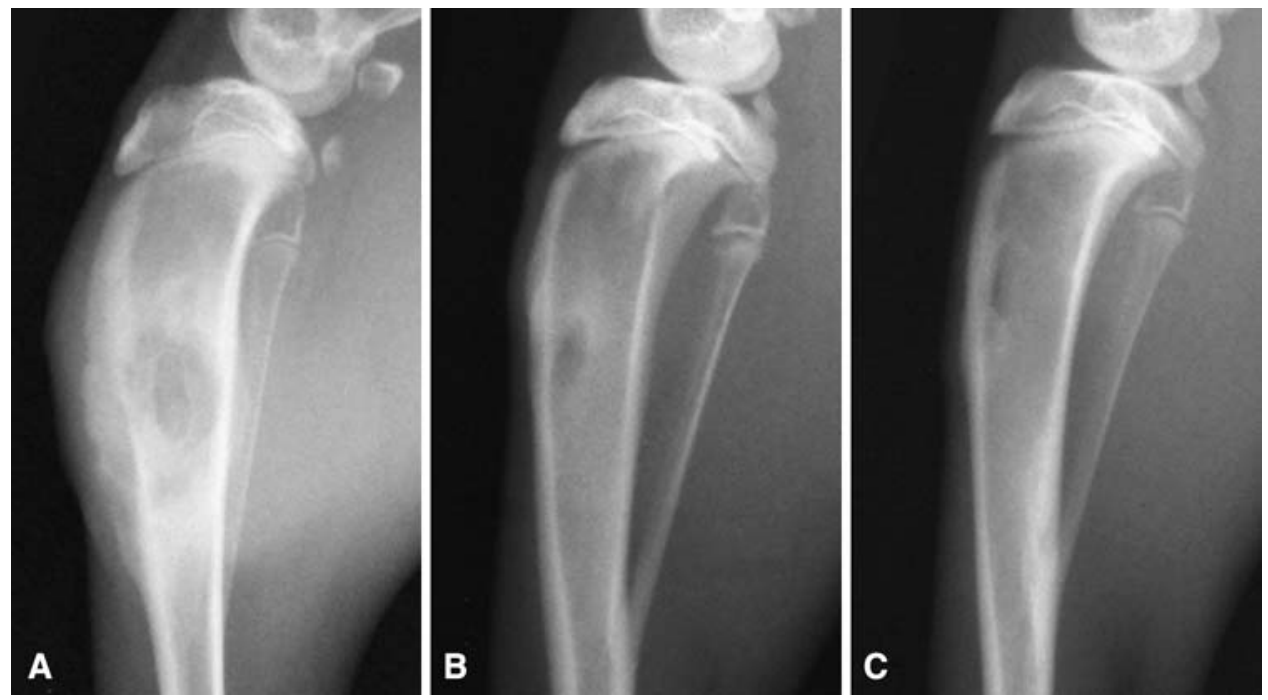

Fig. 2A-C (A) A radiograph taken 4 weeks after surgery shows a bone wax-treated tibia inoculated with Staphylococcus aureus. Clear signs of osteomyelitis can be seen, including bone lysis and periosteal reaction. Bone explants cultured in 5\% TSB showed heavy growth of Staphylococcus aureus. (B) A representative radiograph taken 4

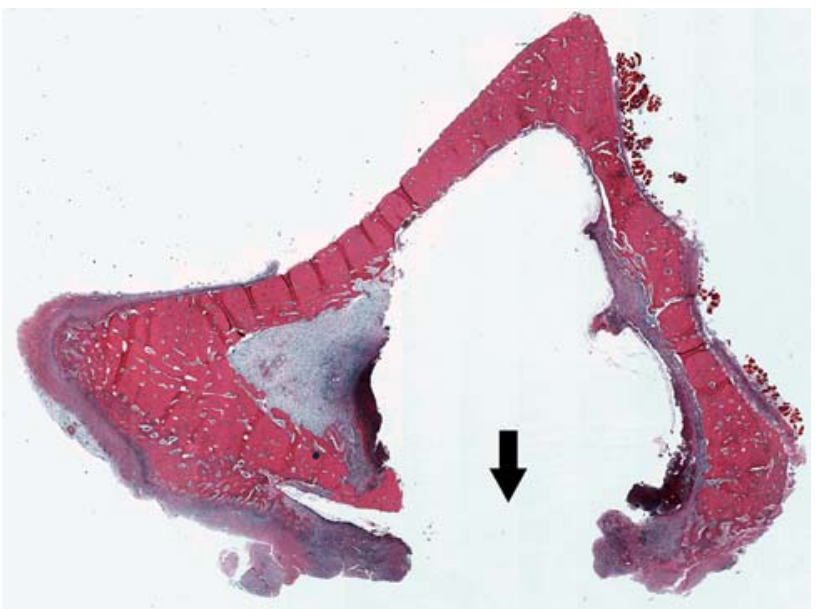

Fig. 3 A cross section of a bone wax-treated tibia at the center of the cortical window shows typical signs of osteomyelitis, including the development of an abscess, the destruction of cortical bone, and periosteal reaction. The cortical window shows no signs of bone healing after 4 weeks (arrow) (Stain, hematoxylin and eosin; original magnification, $\times 5$ ).

Carson's Modern Operative Surgery, the use of bone wax is recommended not for bone hemostasis, but to prevent bone healing and to create a pseudarthrosis as part of an arthroplasty [39]. In various animal studies, bone wax subsequently was shown to inhibit osteogenesis and prevent bone union $[2,5,8,11,12,14,19,20,29,32,36,40]$. Bone wax remains as a foreign body at the site of application indefinitely, and it is known to cause intense foreign body reactions characterized by giant cells, plasma cells, weeks after surgery of a polymer-treated tibia shows no evidence of osteomyelitis and normally healing bone. (C) A representative radiograph of an untreated control tibia also shows no evidence of osteomyelitis and normally healing bone.

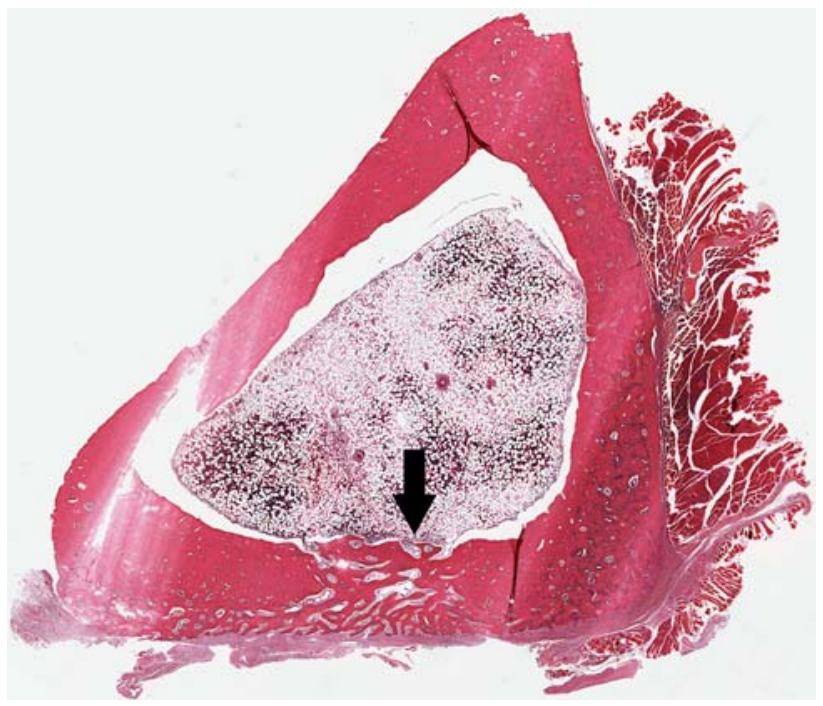

Fig. 4 A cross section of a polymer-treated tibia at the center of the cortical window shows typical normal bone and bone marrow without periosteal reaction. The cortical window is filled with new bone after 4 weeks (arrow) (Stain, hematoxylin and eosin; original magnification, $\times 5)$.

and fibrous tissue [3, 22, 28, 33, 34]. Similar findings also were reported in humans [8, 35, 37]. Bone wax is believed to interfere with osteogenesis, and osteoblasts have been shown to be absent in the presence of a thin layer of bone wax [2]. Suggested appropriate uses for bone wax are prevention of osteosynthesis and osteophyte formation $[2,35]$. 


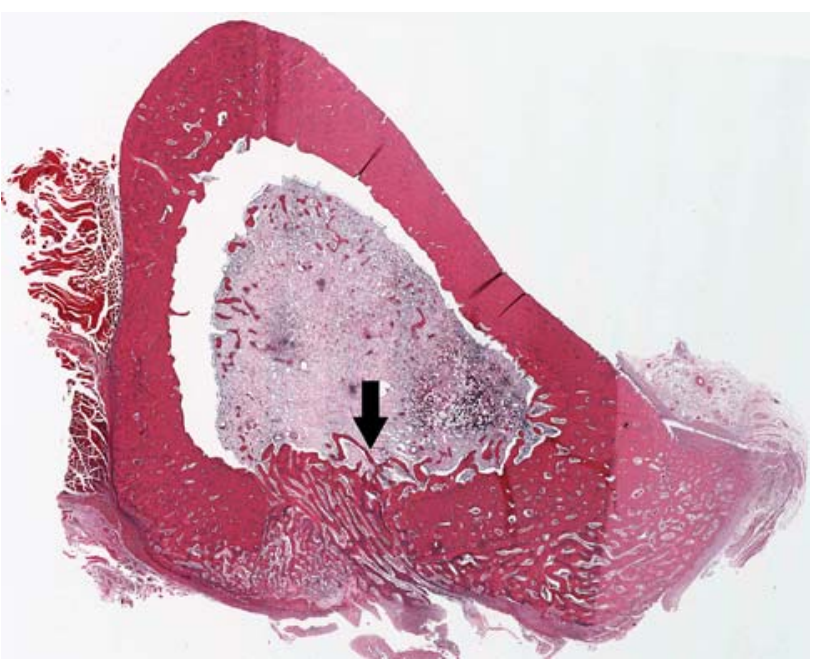

Fig. 5 A cross section of a control tibia at the center of the cortical window also shows normal bone and bone marrow without periosteal reaction. The cortical window is filled with new bone after 4 weeks (arrow) (Stain, hematoxylin and eosin; original magnification, $\times 5$ ).

The inflammatory reactions to bone wax may be a source of postoperative pain. One report described seven patients with intractable pain after the use of bone wax in foot surgery [4]. Five of the patients were pain-free after the bone containing the inflamed bone wax was resected. Clinical reports describing adverse inflammatory reactions to bone wax are common [3-7, 9, 17, 23, 24, 26, 38]. Reactions consist mainly of pain and swelling, often exacerbated by infection.

The alkylene oxide copolymer material used by Wang et al. showed new bone grew within 10 days into a rat femur defect with the polymer and the untreated controls [40]. In contrast, the defects filled with bone wax showed no bone formation 48 days after implantation. The polymer material dissolved from the site of application within 24 to 48 hours, allowing the early phases of bone healing to occur [40]. The polymer material used in our study dissolves in the body and has been shown not to interfere with bone healing or cause inflammation in a sterile environment [41].

In this study, in the presence of bacterial contamination, the use of the polymer material neither increased infection rates nor interfered with bone healing when compared with untreated controls. All of the defects without radiologic evidence of osteomyelitis had normal bone healing. The use of this polymer material in place of bone wax may be another step toward reducing wound complications and the associated morbidity after bone surgery.

Acknowledgments We thank Drs S. Dry and D. Gui from the Tissue Procurement Core Laboratory, Department of Pathology and Laboratory Medicine, UCLA School of Medicine, Los Angeles, CA, for providing the microscopic images of hematoxylin and eosinstained sections.

\section{References}

1. Aimin C, Chunlin H, Juliang B, Tinyin Z, Zhichao D. Antibiotic loaded chitosan bar: an in vitro, in vivo study of a possible treatment for osteomyelitis. Clin Orthop Relat Res. 1999; 366:239-247.

2. Alberius P, Klinge B, Sjögren S. Effects of bone wax on rabbit cranial bone lesions. J Craniomaxillofac Surg. 1987;15:63-67.

3. Allison RT. Foreign body reactions and an associated histological artefact due to bone wax. Br J Biomed Sci. 1994;51:14-17.

4. Anfinsen OG, Sudmann B, Rait M, Bang G, Sudmann E. Complications secondary to the use of standard bone wax in seven patients. J Foot Ankle Surg. 1993;32:505-508.

5. Angelini GD, el-Ghamari FA, Butchart EG. Poststernotomy pseudo-arthrosis due to foreign body reaction to bone wax. Eur J Cardiothorac Surg. 1987;1:129-130.

6. Ates O, Cayli SR, Gürses I. Bone wax can cause foreign body granuloma in the medulla oblongata. Br J Neurosurg. 2004; 18:538-540.

7. Bolger WE, Tadros M, Ellenbogen RG, Judy K, Grady MS. Endoscopic management of cerebrospinal fluid leak associated with the use of bone wax in skull-base surgery. Otolaryngol Head Neck Surg. 2005;132:418-420.

8. Brightmore TG, Hayes P, Humble J, Morgan AD. Haemostasis and healing following median sternotomy. Langenbecks Arch Chir. 1975;(suppl):39-41.

9. Chun PK, Virmani R, Mason TE, Johnson F. Bone wax granuloma causing saphenous vein graft thrombosis. Am Heart $J$. 1988;115:1310-1313.

10. Danielson GK, Dubilier LD, Bryant LR. Use of pluronic F-68 to diminish fat emboli and hemolysis during cardiopulmonary bypass: a controlled clinical study. J Thorac Cardiovasc Surg. 1970;59:178-184.

11. dos Santos Neto FL, Volpon JB. Experimental nonunion in dogs. Clin Orthop Relat Res. 1984;187:260-271.

12. Finn MD, Schow SR, Schneiderman ED. Osseous regeneration in the presence of four common hemostatic agents. J Oral Maxillofac Surg. 1992;50:608-612.

13. Fowler EB, Cuenin MF, Hokett SD, et al. Evaluation of pluronic polyols as carriers for grafting materials: study in rat calvaria defects. J Periodontol. 2002;73:191-197.

14. Geary JR, Kneeland Frantz V. New absorbable hemostatic bone wax: experimental and clinical studies. Ann Surg. 1950; 132:1128-1137.

15. Gibbs L, Kakis A, Weinstein P, Conte JE Jr. Bone wax as a risk factor for surgical-site infection following neurospinal surgery. Infect Control Hosp Epidemiol. 2004;25:346-348.

16. Grindel JM, Jaworski T, Emanuele RM, Culbreth P. Pharmacokinetics of a novel surface-active agent, purified poloxamer 188, in rat, rabbit, dog and man. Biopharm Drug Dispos. 2002;23: 87-103.

17. Hadeishi H, Yasui N, Suzuki A. Mastoid canal and migrated bone wax in the sigmoid sinus: technical report. Neurosurgery. 1995;36:1220-1223 discussion 1223-1224.

18. Horsley V. Antiseptic wax [Letter]. BMJ. 1892;1:1165.

19. Howard TC, Kelley RR. The effect of bone wax on the healing of experimental rat tibial lesions. Clin Orthop Relat Res. 1969; 63:226-232.

20. Ibarrola JL, Bjorenson JE, Austin BP, Gerstein H. Osseous reactions to three hemostatic agents. $J$ Endod. 1985;11:75-83.

21. Jewell RC, Khor SP, Kisor DF, LaCroix KA, Wargin WA. Pharmacokinetics of RheothRx injection in healthy male volunteers. J Pharm Sci. 1997;86:808-812.

22. Johnson P, Fromm D. Effects of bone wax on bacterial clearance. Surgery. 1981;89:206-209. 
23. Katz SE, Rootman J. Adverse effects of bone wax in surgery of the orbit. Ophthal Plast Reconstr Surg. 1996;12:121-126.

24. Kothbauer KF, Jallo GI, Siffert J, Jimenez E, Allen JC, Epstein FJ. Foreign body reaction to hemostatic materials mimicking recurrent brain tumor:report of three cases. J Neurosurg. 2001;95:503-506.

25. Levy ML, Luu T, Meltzer HS, Bennett R, Bruce DA. Bacterial adhesion to surfactant-modified silicone surfaces. Neurosurgery. 2004;54:488-490 discussion 490-491.

26. Low WK, Sim CS. Bone wax foreign body granuloma in the mastoid. ORL J Otorhinolaryngol Relat Spec. 2002;64:38-40.

27. Mehta CR, Patel NR. A network algorithm for performing Fisher's exact test in $\mathrm{r} \times \mathrm{c}$ contingency tables. JASA. 1983; 78(382):427-434.

28. Nelson DR, Buxton TB, Luu QN, Rissing JP. The promotional effect of bone wax on experimental Staphylococcus aureus osteomyelitis. J Thorac Cardiovasc Surg. 1990;99:977-980.

29. Papay FA, Morales L Jr, Ahmed OF, Neth D, Reger S, Zins J. Comparison of ossification of demineralized bone, hydroxyapatite, Gelfoam, and bone wax in cranial defect repair. J Craniofac Surg. 1996;7:347-351.

30. Parker R. Aural pyaemia successfully treated by removing putrid thrombus of jugular vein and lateral sinus. BMJ. 1892;1:1076-1077.

31. Patterson AL, Galloway RH, Baumgartner JC, Barsoum IS. Development of chronic mandibular osteomyelitis in a miniswine model. J Oral Maxillofac Surg. 1993;51:1358-1362.

32. Robicsek F, Masters TN, Littman L, Born GV. The embolization of bone wax from sternotomy incisions. Ann Thorac Surg. 1981;31:357-359.
33. Schonauer C, Tessitore E, Barbagallo G, Albanese V, Moraci A. The use of local agents: bone wax, gelatin, collagen, oxidized cellulose. Eur Spine J. 2004;13(suppl 1):S89-S96.

34. Solheim E, Pinholt EM, Bang G, Sudmann E. Effect of local hemostatics on bone induction in rats: a comparative study of bone wax, fibrin-collagen paste, and bioerodible polyorthoester with and without gentamicin. J Biomed Mater Res. 1992;26: 791-800.

35. Sorrenti SJ, Cumming WJ, Miller D. Reaction of the human tibia to bone wax. Clin Orthop Relat Res. 1984;182:293-296.

36. Sudmann B, Anfinsen OG, Bang G, et al. Assessment in rats of a new bioerodible bone-wax-like polymer. Acta Orthop Scand. 1993;64:336-339.

37. Sudmann B, Bang G, Sudmann E. Histologically verified bone wax (beeswax) granuloma after median sternotomy in 17 of 18 autopsy cases. Pathology. 2006;38:138-141.

38. Verborgt O, Verellen K, Van Thielen F, Deroover M, Verbist L, Borms T. A retroperitoneal tumor as a late complication of the use of bone wax. Acta Orthop Belg. 2000;66:389-391.

39. Verrall PJ. Operation on joints. In: Carson HW, ed. Modern Operative Surgery. Vol 1. London, England: Cassell \& Co; 1924:69.

40. Wang MY, Armstrong JK, Fisher TC, Meiselman HJ, McComb GJ, Levy ML. A new, pluronic-based, bone hemostatic agent that does not impair osteogenesis. Neurosurgery. 2001;49:962-967 discussion 968.

41. Wellisz T, Armstrong JK, Cambridge J, Fisher TC. Ostene, a new water-soluble bone hemostasis agent. J Craniofac Surg. 2006; 17:420-425. 